\title{
Avaliação de risco para infecção HIV em homens que fazem sexo com homens e a contribuição das redes de parceiros sexuais
}

\author{
Risk assessment for HIV infection in men who have sex with \\ men and the contribution of sexual partner networks
}

Raquel Maria Cardoso Torres (https://orcid.org/0000-0003-3052-0106) ${ }^{1}$

Leonardo Soares Bastos (https://orcid.org/0000-0002-1406-0122) 2,3

Marcelo Ferreira da Costa Gomes (https://orcid.org/0000-0003-4693-5402) ${ }^{2}$

Ronaldo Ismerio Moreira (https://orcid.org/0000-0003-2680-4317) ${ }^{4}$

André Reynaldo Santos Périssé (https://orcid.org/0000-0002-5253-5774) ${ }^{5}$

Marly Marques da Cruz (https://orcid.org/0000-0002-4061-474X) ${ }^{5}$

$\overline{{ }^{1} \text { Escola Nacional de Saúde }}$ Pública Sergio Arouca, Fiocruz. R. Leopoldo Bulhões, 1480, Manguinhos, Rio de Janeiro. 21041-210 Rio de Janeiro RJ Brasil. raquelmct@gmail.com ${ }^{2}$ Programa de Computação Científica, Fiocruz. Rio de Janeiro, RJ, Brasil.

${ }^{3}$ Department of Infectious Diseases Epidemiology, London School of Hygiene \& Tropical Medicine. London United Kingdom. ${ }^{4}$ Instituto Nacional de Infectologia, Fiocruz. Rio de Janeiro RJ Brasil.

${ }^{5}$ Escola Nacional de Saúde Pública Sergio Arouca, Departamento de Endemias Samuel Pessoa, Fiocruz. Rio de Janeiro RJ Brasil.
Abstract This study aimed to evaluate the risk of HIV infection in men who have sex with men (MSM) by developing an index that considers sex partner networks. The index variables were age, ethnicity/skin color, schooling, relationship type, condom use in receptive and insertive relationships, self-perception of the possibility of HIV infection, sexually transmitted infections, and rapid HIV testing results. We used data from a cross-sectional MSM egocentric network survey conducted in Rio de Janeiro between 2014 and 2015. The initial research volunteer is called ego, each partner is called alter, and each pair of people in a relationship is called the dyad. Multiple logistic regression was used to define the coefficients of the equations for the elaboration of the indices. The index ranged from 0 to 1 ; the closer to 1 , the higher the risk of HIV infection. HIV prevalence was $13.9 \%$ among egos. The mean egos index with an HIV-reactive test was 57\% higher than non-reactive, and the same profile was observed in the index values of dyads. The index allowed the incorporation of network data through the dyads and contributed to the identification of individuals with a higher likelihood of acquiring HIV. Key words HIV, Male homosexuality, Social network, Sexual behavior, Risk indicator
Resumo O objetivo do estudo foi avaliar o risco de infecção por HIV em homens que fazem sexo com homens (HSH) a partir do desenvolvimento de um indice que considere as redes de parceiros sexuais. As variáveis do indice foram faixa etária, raça/cor, escolaridade, tipo de relacionamento, uso de preservativo em relações receptivas e insertivas, autopercepção da chance de se infectar pelo HIV, história de infecções sexualmente transmissíveis, além dos resultados dos testes rápidos para HIV. Foram utilizados dados de uma pesquisa de rede egocêntricas $\mathrm{HSH}$, com desenho transversal, realizada no Rio de Janeiro entre 2014 e 2015. O voluntário inicial da pesquisa é denominado ego, cada parceiro é alter, e cada par de pessoas em um relacionamento é a díade. Utilizou-se regressão logística múltipla para definição dos coeficientes das equações para elaboração dos indices. O indice variou de 0 a 1, quanto mais próximo de 1, maior o risco de infecção por HIV. A prevalência de HIV dos egos foi de 13,9\%. A média do índice dos egos com teste HIV reagente foi 57\% maior do que aqueles não reagentes, o mesmo perfil foi observado nos valores dos indices das díades. O indice permitiu incorporar os dados das redes por meio das díades e contribuiu para a identificação de indivíduos com maior chance de aquisição do HIV. Palavras-chave HIV, Homossexualidade masculina, Rede social, Comportamento sexual, Indicador de risco 


\section{Introdução}

Desde a década de 1990, inúmeros investimentos e avanços ocorreram para a deteç̧ão e tratamento precoce do HIV/Aids no Brasil. Entretanto, ainda se observa uma elevada prevalência que afeta principalmente populações específicas, como os usuários de drogas, os homens que fazem sexo com homens (HSH) e as(os) profissionais do sexo $^{1-4}$. Os HSH apresentam uma das maiores prevalências de infecção pelo HIV entre essas populações e diversos estudos brasileiros indicam valores em torno de $10 \%$ ou superior ${ }^{5-9}$.

Diante da complexa dinâmica de transmissão do HIV entre os HSH, destaca-se uma forte associação entre a infecção por HIV e práticas e comportamentos sexuais, uso de preservativo, tipo e número de parceiros sexuais e uso de drogas/ álcool ${ }^{10-13}$. Entretanto, a maioria desses estudos que avaliam as vulnerabilidades e fatores de risco para HIV/Aids dão mais ênfase ao componente individual.

Nesse contexto, estudos que consideram outros aspectos, como a rede social, têm demonstrado grande relevância na compreensão da disseminação do HIV entre os HSH. A estrutura das relações sociais em uma rede influencia o conteúdo dessas relações, de forma que a rede de parceiros sexuais pode influenciar no risco de adquirir uma infecção sexualmente transmissível (IST). Portanto, incorporar aspectos de rede nos estudos adiciona informação para além das características individuais ${ }^{14-20}$.

A análise da rede social permite avaliar a influência das conexões entre as pessoas no processo de transmissão de uma dada doença, ou seja, considera as relações e como elas afetam a dinâmica de indivíduos e grupos. Dessa forma, a análise de redes possibilita transformar os dados coletados em nível individual para dados de suas interações em grupo ${ }^{21-23}$. Entre os desenhos de estudos de redes sociais, destaca-se nesse artigo o tipo egocêntrico, também denominada de rede pessoal ou local. A análise do tipo egocêntrica pode ser feita por meio das díades. Uma díade é definida como um par de pessoas em um relacionamento, e por isso, é a unidade principal dos dados de uma rede egocêntrica. A análise das díades em uma rede egocêntrica viabiliza estudar a influência da rede no comportamento dos indivíduos em situações de saúde, como a aquisição e transmissão da infecção do HIV ${ }^{24}$.

Além da incorporação das redes sociais nas análises de risco de aquisição da infecção por HIV, é fundamental encontrar uma forma prática e acessível de mensurar aspectos individuais e de rede que possam ser utilizados para o desenvolvimento de políticas públicas. Nesse sentido, destaca-se a construção de índices que permitem sintetizar em um valor único o risco para o HIV. Estudo brasileiro recente demonstrou como um índice é capaz de sumarizar diversos aspectos ${ }^{7}$. Outros estudos internacionais também trabalharam em uma proposta de síntese ou escala de comportamento de risco para infecção pelo HIV a partir da combinação de diferentes características ${ }^{25,26}$.

Diante do exposto, este trabalho teve como objetivo avaliar o risco de infecção por HIV em HSH a partir do desenvolvimento de um índice que considere as redes de parceiros sexuais. Tal objetivo se justifica pela tendência de crescimento da infecção pelo HIV, principalmente entre os HSH mais jovens, e pela necessidade de aprimoramento das estratégias de prevenção e tratamento precoce nesse grupo.

\section{Método}

Foram utilizados os dados de um estudo de rede egocêntrica com desenho transversal realizado no município do Rio de Janeiro em uma população de HSH denominado "Uso da análise de redes sociais para o estudo de fatores associados à aquisição do HIV entre homens que fazem sexo com homens". No desenho de rede egocêntrica, os dados sobre cada indivíduo são coletados e na sequência são levantados os dados acerca daqueles indivíduos com que eles tiveram algum tipo de interação ou relacionamento ${ }^{27}$.

\section{Recrutamento e coleta de dados}

Os participantes elegíveis para a pesquisa foram os homens com idade igual ou superior a 18 anos que tenham relatado relações sexuais com homens nos últimos seis meses. Além disso, também foi critério de elegibilidade que o voluntário fosse não reagente para o HIV ou desconhecesse seu status sorológico. O recrutamento foi realizado de três formas: 1) convite pessoalmente às pessoas que estivessem visitando algum dos locais de coleta de dados da pesquisa, seja para alguma atividade, consulta médica ou apenas conhecendo a unidade; 2) convite pessoalmente às pessoas que estavam passando em ruas próximas a essas unidades; 3 ) convite por meio de recrutadores, que entregavam um cartão com informações da pesquisa, local e horário para responder ao questionário, às pessoas em locais seleciona- 
dos previamente onde havia afluxo da população de HSH, como bares, festas, boates e saunas em diferentes bairros do Rio de Janeiro. Os locais de coleta de dados foram: duas organizações não governamentais (ONGs) e uma unidade da Estratégia de Saúde da Família (ESF) localizadas na região central do município do Rio de Janeiro. A pesquisa foi aprovada pelo Comitê de Ética em Pesquisa da Escola Nacional de Saúde Pública Sergio Arouca (ENSP/Fiocruz/Brasil).

A amostra da população de HSH do estudo foi calculada a partir da hipótese de que diversos fatores importantes na transmissão do HIV serão maiores em voluntários HIV reagentes quando comparados aos voluntários HIV não reagentes. Assim, optou-se por estimar o menor tamanho do efeito detectável baseado em tamanhos amostrais fixos para formar um grupo de HIV reagentes de 100 voluntários, considerando uma média de prevalência de HIV na população $\mathrm{HSH}$ de $15 \%{ }^{2,4,6,28}$. Então a amostra total final foi de 700 indivíduos $\mathrm{HSH}$, valor de alfa igual a 0,05 , poder de 0,8 e alocação não balanceada (1:6).

Neste estudo, o voluntário inicial da pesquisa foi denominado ego, e cada integrante de sua rede de relacionamento, fosse sexual ou de amizade, foram denominados alters. Além disso, todas as questões sobre parceiros sexuais ou amigos foram respondidas pelo ego. Os egos responderam sozinhos aos questionários computadorizados online utilizando o programa SurveyGizmo em salas individualizadas nos locais da pesquisa. Foram usados quatro questionários diferentes, sendo o primeiro com perguntas específicas sobre si mesmo, como características sociodemográficas, história clínica de IST, práticas e comportamentos sexuais. Ao final desse questionário, solicitava-se ao voluntário que listasse até no máximo cinco pessoas com que ele tivera relações sexuais nos últimos seis meses. A partir dessa lista, ele respondeu a outro questionário específico para cada parceiro sexual listado. O mesmo foi realizado para a rede social de amizade, em que o voluntário listou até cinco pessoas com que ele teve amizade nos últimos seis meses e então respondeu a outro questionário específico para cada amigo listado. Por fim, o voluntário respondeu sobre as relações existentes entre os parceiros sexuais e amigos listados nos questionários anteriores. Para que lembrasse de todos os seus parceiros, utilizou-se uma técnica denominada namegenerator, empregada para aumentar a recordação de parceiros sexuais ${ }^{29,30}$.

Depois de responder aos questionários, os voluntários (egos) realizaram o teste para o HIV.
Foram usados dois tipos de teste, dependendo do local da pesquisa: teste rápido de diagnóstico por exame laboratorial de sangue e teste rápido por fluído oral para triagem de detecção do HIV que, quando davam resultado reagente, necessitavam do confirmatório. Os dados sobre rede social de amizade e a matriz de relação entre os parceiros sexuais e amigos não foram utilizados neste estudo.

A partir dos quatro questionários, foram gerados quatro bancos de dados, dos quais neste estudo foram utilizados somente dois, referentes aos seguintes questionários: 1) dados do ego com questões sobre si próprios e 2) dados de cada parceiro sexual de sua rede.

\section{Construção do índice de risco para infecção por HIV}

O desenvolvimento do índice de risco para infecção por HIV que considera a influência da rede de parceiros sexuais entre os $\mathrm{HSH}$ se deu em três etapas. Primeiro foi calculado um índice que sintetizou em um único valor as características, práticas e comportamentos sexuais do indivíduo (ego). Em seguida foi calculado outro índice que sintetizou as mesmas informações dos parceiros sexuais (alters). Por fim, o resultado desses dois índices foi comparado com o intuito de verificar o quanto um índice que inclui a informação da rede de parceiros sexuais influenciou no risco de aquisição do HIV.

A seleção das variáveis que compuseram o índice foi realizada com base na revisão da literatura, buscando identificar aspectos que apresentassem associação com a infecção por HIV ${ }^{10,15,31-35}$, e por meio de debate com especialistas em pesquisas em HIV/Aids no Brasil. Além disso, para a construção desses dois índices foi necessário utilizar variáveis que representassem situações semelhantes em ambos os questionários de egos e alters. Assim, as variáveis incluídas no estudo foram: faixa etária (<= 30 anos e $>30$ anos), raça/cor (negro e não negro), escolaridade (nenhuma até nível médio incompleto e nível médio completo ou mais), tipo de relacionamento (casado/amigado e solteiro/separado), uso de camisinha em sexo anal receptivo com parceiro regular ou casual, uso de camisinha em sexo anal insertivo com parceiro regular ou casual, autopercepção da chance de se infectar com HIV (pontuação de 0 a 2, 3 a 5 e 6 a 10), ter tido alguma IST e resultado do teste HIV dos egos.

Para responder às questões de acordo com o tipo de parceria sexual, foram incluídas no ques- 
tionário as seguintes definições: "parceiros regulares (fixos)" foram aqueles com quem ele teve relação sexual e teve um caso, namoro, encontros frequentes, casamento ou qualquer tipo de envolvimento; "parceiros casuais" foram aqueles com quem ele teve relação sexual sem marcar outros encontros ou sem estabelecer qualquer compromisso de continuidade na relação.

Novas variáveis foram construídas a partir da agregação ou combinação de categorias. A variável raça/cor teve as opções "negro" e "pardo" agrupadas na categoria "negro", enquanto "branco" e "amarelo/indígena" ficaram agrupadas na categoria "não negro". Nessa questão foi adicionada o preenchimento da variável de texto para aqueles que marcaram a opção "outra": a resposta "morena(o)" foi agrupada na categoria "negro". A variável IST foi construída a partir de perguntas específicas para doenças no questionário dos egos e foram organizadas da seguinte maneira: quem respondeu ter sido diagnosticado com sífilis, gonorreia, clamídia ou outra doença transmitida pelo sexo foi agrupado em "sim teve IST", e o restante como "não teve IST". Entretanto, no questionário dos alters foi apenas uma pergunta direta, para a qual não foi necessário nenhum agrupamento, pois foi perguntado somente se o ego sabia se aquele parceiro teve alguma IST (sim ou não).

A questão da utilização de preservativo teve as opções agrupadas da seguinte forma: "sim, usou camisinha" apenas quando responderam ter utilizado preservativo sempre, em todas as relações sexuais; e "não usou camisinha" para as demais opções. A variável uso de camisinha em relação sexual anal (insertiva ou receptiva), segundo o tipo de parceria (regular ou casual), foi uma junção de três variáveis: se realizou essa prática sexual nos últimos seis meses; frequência de utilização do preservativo nesse tipo de prática sexual; e se teve parceiro regular ou casual nos últimos seis meses. Por fim, a variável uso de camisinha segundo a prática sexual e o tipo de parceiro foi categorizada da seguinte forma: "sim, usou camisinha"; "não usou camisinha" e "não fez sexo anal (receptivo ou insertivo)/não teve parceiro (regular ou casual)".

As variáveis quantitativas contínuas referentes a faixa etária e autopercepção da chance de se infectar com HIV foram categorizadas de acordo com a distribuição de frequência dos dados.

A primeira etapa para o cálculo do índice dos egos foi a realização de regressão logística ${ }^{36}$, tendo como variável dependente o resultado do teste de HIV dos egos, e todas as demais como variá- veis independentes gerando a seguinte equação, conhecida por função logística:

$$
\mathrm{P}\left(\mathrm{Y}_{i}=1\right)=\frac{1}{1+\mathrm{e}_{i}^{-\mathrm{x} T \beta}} \quad \text { (Equação 1) }
$$

Onde $Y_{i}$ é o status do teste de HIV para o participante $i$, assumindo o valor 1 se o teste for reagente e 0 caso contrário, $\mathrm{x}_{i}$ são as variáveis independentes do participante $i$, e $\beta$ são os coeficientes de regressão da equação (1). Os coeficientes foram estimados com os dados dos egos, ajustando um modelo de regressão logística. E ao aplicar a equação (1) para cada ego, encontrou-se um valor único denominado "índice ego".

Para a segunda etapa, que envolveu a construção do índice de parceiros sexuais, essa equação (1) foi aplicada aos dados dos alters, gerando outro valor único para cada parceiro sexual, que foi denominado "índice alter". O valor final dos índices variou de 0 a 1 , sendo que os valores mais próximos de 1 significam maior risco de infecção pelo HIV. O índice alter foi considerado como o índice de risco para infecção, indicando a influência da rede de parceiros sexuais entre os HSH.

$\mathrm{Na}$ terceira etapa, foram comparados os resultados entre os índices dos egos e dos alters de acordo com o resultado do teste de HIV dos egos. Por essa razão, os bancos de dados de egos e alters, com os respectivos resultados dos índices, foram relacionados a partir de uma variávelchave com código de identificação para cada ego, formando um único banco. Esse procedimento se fez necessário pelo fato de que o teste rápido para HIV foi aplicado apenas aos egos. Assim, para a análise dos índices dos alters foram consideradas as díades entre cada parceiro sexual e o ego, permitindo incluir o resultado do teste HIV de seus respectivos egos.

Por fim, foi construída a variável "diferença dos índices", que representou o resultado da subtração dos valores dos índices de egos e alters. Se o resultado foi positivo, significou que aquele alter específico apresentou menor risco do que o ego, se o valor foi negativo, então aquele alter teve maior risco do que o ego, e se foi nulo significa que egos e alters apresentaram riscos iguais, ou seja, índices com os mesmos valores. Ao comparar os resultados dos índices de egos e alters, buscou-se verificar a influência da rede de parceiros sexuais para o risco de aquisição da infecção por HIV. A verificação da diferença entre os índices de egos e alters é uma indicação de que utilizar dados de parceiros sexuais de um estudo de rede egocêntrica pode adicionar informações relevantes à análise de risco para infecção por HIV. 


\section{Resultados}

O questionário foi preenchido por 341 indivíduos (egos), o que representou $49 \%$ da amostra planejada 9 . Entretanto, para a construção da equação que gerou os índices não poderia haver variáveis sem informação, por isso foram excluídos aqueles que não preencheram alguma das perguntas/variáveis utilizadas neste estudo. Além disso, entre os voluntários (egos), dois recusaram realizar o teste de HIV ao final do questionário e foram excluídos. Assim, do total de egos, 331 $(97,1 \%)$ tinham informações completas para todas as variáveis utilizadas na construção do índice e foram incluídos na análise. Esses egos responderam ao questionário de 622 parceiros sexuais, sendo necessário excluir alguns registros pelas razões descritas anteriormente, assim, ao final foram 588 alters (94,5\%).

A prevalência HIV entre os egos que realizaram o teste de HIV foi de 13,9\% (46 em 331). Aproximadamente 11\% (65 em 588) das díades foram provenientes de egos HIV reagentes. A análise descritiva dos egos foi apresentada em publicação anterior'. De forma resumida, o grupo total de egos (341) foi em sua maioria pardos, com elevado nível de escolaridade, solteiros ou separados e média de idade de 30,6 anos. O relato de IST foi baixo entre os egos, mas a maior parte deles mencionou ter realizado teste de HIV outras vezes anteriormente à pesquisa. Em média os egos informaram dois parceiros sexuais regulares e cinco parceiros casuais nos últimos seis meses. Entretanto, a média de parceiros sexuais, sobre os quais os egos responderam a um questionário específico para cada um deles, foi de 1,9 parceiro, sendo 1,1 para os regulares e 2,0 para os casuais. As características dos alters também já foram descritas em publicação anterior ${ }^{9}$ e os mesmos apresentaram média de idade de 28 anos e, em

sua maioria, elevado nível de escolaridade e de raça/cor negro. Os egos declararam que a maioria dos alters representavam relações não regulares e que os mesmos mantinham parcerias simultâneas além da díade ego-alter?.

As estimativas dos coeficientes da equação (1) via ajuste da regressão logística nos dados dos egos e utilizados para a construção dos índices dos alters estão apresentadas na Tabela 1. Vale notar que estamos ignorando a incerteza associada às estimativas dos coeficientes, uma vez que incluímos todas as variáveis em comum dos conjuntos de dados de egos e alters que estão associados à infecção pelo HIV, de acordo com a literatura e o debate com especialistas.

A Tabela 2 mostra a estatística descritiva dos índices de egos e alters segundo resultado do teste de HIV. Observa-se que a média do índice de todos os egos foi de 0,14 , entretanto os egos com teste HIV reagente $(0,27)$ apresentaram índice $57 \%$ maior do que aqueles não reagentes $(0,12)$. Para a mediana, percentis 25 e 75, os valores dos índices de egos com teste HIV reagentes foram superiores aos não reagentes. É importante observar que $25 \%$ dos egos HIV reagentes possuíam índices acima de 0,41 , e o valor máximo do índice nesse grupo foi de 0,89 (Tabela 2 ).

Entre os alters, a média e a mediana dos índices foram 0,14 e 0,08, respectivamente. Também foi verificada uma média dos índices levemente superior entre aqueles da rede de egos com teste HIV reagente $(0,19)$ em relação aos não reagentes $(0,14), 24 \%$ acima. Os valores dos índices mínimo e máximo foram de 0,004 e 0,85 , respectivamente (Tabela 2).

Os valores dos índices de egos e alters de forma comparativa se encontram na Tabela 3. Entre as díades com egos reagentes para HIV, observouse que a maioria dos alters estavam com valores de índice de risco para HIV inferior aos dos egos $(73,8 \%)$. Entre as díades com egos não reagentes para HIV, a diferença entre os valores dos índices de egos e alters ficou em torno de 50\% (Tabela 3).

\section{Discussão}

Os estudos brasileiros que investigam a influência da rede de parceiros sexuais para a infecção pelo HIV na população de HSH são escassos ${ }^{18,37}$. Isso implica o não acesso a informações qualificadas sobre as redes de parcerias sexuais e o consequente uso dessas informações para a melhoria das estratégias de prevenção para grupos sociais mais vulneráveis, como os HSH. Assim, este 
Tabela 1. Coeficientes estimados para regressão logística ajustada para infecção por HIV dos egos, 2015, Rio de Janeiro, Brasil.

\begin{tabular}{|c|c|c|c|}
\hline & $\begin{array}{c}\text { Coeficiente } \\
\text { estimado }\end{array}$ & $\begin{array}{c}\text { Intervalo de } \\
\text { confiança de } 95 \% \\
\end{array}$ & valor-p \\
\hline Intercepto & $-2,936$ & $(-4,678 ;-1,252)$ & 0,001 \\
\hline $\begin{array}{l}\text { Faixa etária: ref. }<30 \text { anos; } \\
\qquad>=30 \text { anos }\end{array}$ & $-0,272$ & $(-1,027 ;-0,457)$ & 0,470 \\
\hline $\begin{array}{l}\text { Raça/cor: ref. não negro; } \\
\text { negro }\end{array}$ & 0,374 & $(-0,371 ; 1,161)$ & 0,336 \\
\hline $\begin{array}{l}\text { Escolaridade: ref. }<2^{\circ} \text { grau; } \\
\qquad>=2^{\circ} \text { grau }\end{array}$ & $-0,108$ & $(-1,081 ; 0,974)$ & 0,834 \\
\hline $\begin{array}{l}\text { Relacionamento: ref. casado/amigado; } \\
\text { solteiro/separado }\end{array}$ & $-0,655$ & $(-1,458 ; 0,179)$ & 0,114 \\
\hline $\begin{array}{l}\text { Uso camisinha sexo passivo com parceiro } \\
\text { REGULAR: ref. passivo SEM camisinha; } \\
\text { passivo COM camisinha; } \\
\text { não sexo ou parceiro regular }\end{array}$ & $\begin{array}{l}0,602 \\
0,671\end{array}$ & $\begin{array}{l}(-0,778 ; 2,010) \\
(-0,417 ; 1,848)\end{array}$ & $\begin{array}{l}0,395 \\
0,242\end{array}$ \\
\hline $\begin{array}{l}\text { Uso camisinha sexo passivo com parceiro } \\
\text { CASUAL: ref. passivo SEM camisinha; } \\
\text { passivo COM camisinha; } \\
\text { não sexo ou parceiro regular }\end{array}$ & $\begin{array}{l}-1,548 \\
-2,116\end{array}$ & $\begin{array}{l}(-3,043 ;-0,105) \\
(-3,580 ;-0,725)\end{array}$ & $\begin{array}{l}0,037 \\
0,003\end{array}$ \\
\hline $\begin{array}{l}\text { Uso camisinha sexo ativo com parceiro } \\
\text { REGULAR: ref. ativo SEM camisinha; } \\
\text { ativo COM camisinha; } \\
\text { não sexo ou parceiro regular }\end{array}$ & $\begin{array}{l}-0,107 \\
-0,031\end{array}$ & $\begin{array}{l}(-1,517 ; 1,307) \\
(-1,102 ; 1,079)\end{array}$ & $\begin{array}{l}0,881 \\
0,955\end{array}$ \\
\hline $\begin{array}{l}\text { Uso camisinha sexo ativo com parceiro } \\
\text { CASUAL: ref. ativo SEM camisinha; } \\
\text { ativo COM camisinha; } \\
\text { não sexo ou parceiro regular }\end{array}$ & $\begin{array}{l}0,648 \\
2,128\end{array}$ & $\begin{array}{r}(-0,957 ; 2,232) \\
(0,633 ; 3,814)\end{array}$ & $\begin{array}{l}0,435 \\
0,008\end{array}$ \\
\hline $\begin{array}{l}\text { Chance de aquisição HIV: ref. } 0 \text { a } 2 \text { pontos; } \\
\qquad \begin{array}{l}3 \text { a } 5 \text { pontos; } \\
6 \text { a } 10 \text { pontos }\end{array}\end{array}$ & $\begin{array}{l}1,398 \\
2,320\end{array}$ & $\begin{array}{l}(0,597 ; 2,249) \\
(1,320 ; 3,359)\end{array}$ & $\begin{array}{r}0,001 \\
<0,001\end{array}$ \\
\hline $\begin{array}{l}\text { IST: ref. não; } \\
\quad \operatorname{sim}\end{array}$ & 0,636 & $(-0,121 ; 1,391)$ & 0,098 \\
\hline
\end{tabular}

Fonte: Elaborado pelos autores.

estudo buscou considerar a contribuição que as informações da rede de parceiros sexuais podem fornecer na compreensão da dinâmica de transmissão da infecção por HIV.

No presente artigo, optou-se por adotar o estudo de rede egocêntrica, em que apenas o recrutamento dos egos é realizado, com as informações de parcerias sexuais (alters) sendo respondidas pelos próprios egos ${ }^{18,38}$. Tal característica aumenta o tamanho da amostra de análise sem necessariamente tornar mais complexo e encarecer o estudo. Outro aspecto relevante nos estudos de redes de relacionamento que foi utilizado neste trabalho foi a análise das díades, ou seja, os pares formados por ego e alter, para relacionar as características dos parceiros sexuais (alters) com o resultado do teste HIV de seus respectivos $\operatorname{egos}^{24,38,39}$. Dessa forma, permitiu incorporar ao computo geral do risco pessoal de cada ego os fatores de risco dos parceiros (alters) e suas interações com os egos. Hedberg ${ }^{40}$ demostrou como as redes sociais são construídas com díades e como os resultados das relações em díade são influenciadas pela qualidade das díades e da rede.

Estudos de rede egocêntrica ainda são raros no Brasil ${ }^{9,18}$, mas têm sido utilizados em outros países. Rodriguez-Hart e colaboradores ${ }^{19}$ realizaram um estudo de rede egocêntrica na Nigéria para analisar práticas de serosorting de acordo com os estados sorológicos de HIV dos voluntários. Os autores identificaram que as díades soroconcordantes apresentaram riscos menores para infecção por HIV. 
Tabela 2. Estatística descritiva dos índices de risco para infecção HIV para os egos e alters segundo resultado HIV das díades, 2015, Rio de Janeiro, Brasil.

\begin{tabular}{lccc}
\hline & $\begin{array}{c}\text { Resultado do teste HIV } \\
\text { das díades }\end{array}$ & \\
\cline { 2 - 3 } & Reagente & $\begin{array}{c}\text { Não } \\
\text { reagente }\end{array}$ & \\
\hline Egos $(\mathrm{n}=331)$ & & & \\
Média & 0,27 & 0,12 & 0,14 \\
Mediana & 0,22 & 0,06 & 0,08 \\
P25* & 0,13 & 0,04 & 0,04 \\
P75 & 0,41 & 0,14 & 0,18 \\
Alters $(\mathrm{n}=588)$ & & & \\
Média & 0,19 & 0,14 & 0,14 \\
Mediana & 0,11 & 0,08 & 0,08 \\
P25 & 0,04 & 0,04 & 0,04 \\
P75 & 0,27 & 0,19 & 0,19 \\
${ }^{*}$ Percentil 25; ${ }^{* *}$ Percentil 75 & &
\end{tabular}

Fonte: Elaborado pelos autores.

Os dados da rede de parceiros sexuais encontrados no estudo atual foram incorporados na construção de um índice de risco de infecção por HIV entre HSH. Acredita-se que esse método seja adequado por ser uma forma simples e prática de sumariar diversos aspectos individuais (ego) e de rede (alters) num único valor para avaliar o risco de infecção pelo HIV. O índice permitiu discriminar aqueles indivíduos que estavam sob maior risco de aquisição da infecção HIV. Além disso, o método proposto para construção do índice teve variáveis incluídas em um modelo de regressão logística definidas com base na literatura e em debate com especialistas. Tal método possibilitou resumir em um valor único as características, práticas e comportamentos de risco abordados na literatura como associados à aquisição do HIV dos diversos atores das redes.

A proposta de sintetizar em um valor único variáveis para avaliar o risco de infecção por HIV foi verificada em outros estudos ${ }^{7,25,26}$. Embora não seja possível uma comparação direta dos resultados por conta da diversidade de métodos utilizados, pode-se destacar algumas semelhanças com os resultados encontrados no estudo atual. Mattson e colaboradores ${ }^{25}$ desenvolveram uma escala para medir o comportamento de risco sexual entre homens no Quênia a partir da combinação de 18 variáveis. Entre os resultados desse estudo, destaca-se a associação estatisticamente significativa entre a escala de risco proposta pelos autores e a aquisição de IST e HIV.

Outro estudo brasileiro conduzido por Rocha e colaboradores ${ }^{7}$ desenvolveu um escore de comportamento de risco para infecção por HIV para a população de $\mathrm{HSH}$ e encontrou baixa pontuação. Nessa pesquisa, a pontuação variava de $0 \mathrm{a}$ 48 pontos, com a média de pontos entre indivíduos HSH sendo de 5,7. De acordo com a classificação adotada no estudo, $26 \%$ e $54,2 \%$ foram classificados como baixo ( 0 a 2 pontos) e médio ( 3 a 8 pontos) comportamento de risco para HIV,

Tabela 3. Frequência e proporção da comparação entre os índices de risco para infecção HIV dos egos e alters segundo resultado HIV das díades, 2015, Rio de Janeiro, Brasil.

\begin{tabular}{|c|c|c|c|}
\hline & \multicolumn{3}{|c|}{ Resultado do teste HIV das díades } \\
\hline & Reagente & Não reagente & Total \\
\hline & $(n=65) \quad \%(n)$ & $(n=523) \quad \%(n)$ & $(\mathbf{n}=\mathbf{5 8 8}) \quad \%(\mathbf{n})$ \\
\hline Índice do alter maior do que do ego & $26,2(17)$ & $50,1(262)$ & $47,4(279)$ \\
\hline Índice de egos e alters iguais & 0 & $1,1(6)$ & $1,0(6)$ \\
\hline Índice de alter menor do que do ego & $73,8(48)$ & $48,8(255)$ & $51,5(303)$ \\
\hline
\end{tabular}

Fonte: Elaborado pelos autores. 
respectivamente. Entretanto, os pesquisadores identificaram que o escore de alto risco foi de $28,8 \%$ para os reagentes para HIV, o que representou proporção superior à encontrada entre os indivíduos HIV não reagentes (19,7\%).

Assim como a pesquisa de Rocha e colaboradores $^{7}$, o presente estudo também encontrou valores baixos para os índices de risco, embora o método de construção do escore e a forma de amostragem tenham sido diferentes. Apesar dos valores médios e das medianas dos índices entre os egos e as díades HIV reagentes terem sido baixos, ainda assim foram superiores aos encontrados em egos e díades HIV não reagentes, o que demostra que o índice permitiu identificar os indivíduos com maior risco. Em relação à incorporação dos dados de rede de parceiros sexuais para construção do índice de risco de infecção HIV, observou-se que os valores dos índices dosegos foram superiores aos índices dos alters entre as díades de egos HIV reagentes. Tal fato pode significar que os egos estão mais envolvidos em comportamentos de risco para HIV e que possuem uma percepção de baixo risco em relação a sua rede de parceiros sexuais. Ou seja, o índice conseguiu identificar que os voluntários entrevistados (egos) possuíam características de risco para HIV mas acreditavam que seus parceiros (alters) apresentavam baixo comportamento de risco sexual, resultando em valores mais baixos entre os parceiros.

O estudo apresentou resultados relevantes diante do contexto de risco de infecção por HIV entre os HSH. Entretanto, a análise dos resultados deve ser vista levando em consideração suas limitações. Uma das grandes dificuldades da pesquisa foi acessar a população de $\mathrm{HSH}$, o que tornou necessário fazer modificações no recrutamento dos participantes ao longo do desenvolvimento do estudo. Mesmo assim não foi possível atingir o total da amostra planejada, o que pode ter reduzido o poder do estudo para identificar algumas variáveis importantes. Cabe salientar que os HSH são considerados uma população de difícil acesso, como já descrito em pesquisas anteriores ${ }^{15,43,44}$. Soma-se a isso o fato de termos selecionado os voluntários entre aqueles que frequentam locais específicos, como ONGs e uma ESF voltada à população HSH e trans. Tal fato pode ter gerado um viés de seleção no estudo, em que o grupo selecionado pode representar aqueles que têm maior preocupação com o bem-estar físico e psicológico e/ou indivíduos mais engajados em atividades da população LGBT. Outra limitação identificada no estudo diz respeito ao tempo de preenchimento do questionário completo, cerca de uma hora, o que limitava o número de voluntários que poderiam respondê-lo na íntegra por dia.

Foram excluídos do estudo os indivíduos sabidamente HIV reagentes, uma vez que a proposta de construir o índice de risco para HIV foi justamente o de aferir o risco anterior à soroconversão. Apesar de existir a possibilidade de viés de informação/memória devido às questões comportamentais e específicas de cada um de seus parceiros sexuais se referirem a aspectos nos últimos seis meses, ressalta-se que o período adotado foi inferior ao utilizado em outros estudos. Para minimizar esse viés, optou-se por utilizar técnicas desenvolvidas de namegenerator ${ }^{29,30}$ que contribuíram para a lembrança dos aspectos das parcerias sexuais.

Embora a pesquisa tenha se deparado com dificuldades de acesso à população de HSH e limitações na coleta de dados, destaca-se a importância de ter atingido $49 \%$ da amostra planejada de uma população de difícil acesso. Além disso, os dados indicam uma amostra diferenciada e com alto risco para aquisição do HIV. Os achados evidenciaram que a população de HSH estudada está sob elevado risco de infecção HIV e que um estudo de rede do tipo egocêntrica pode contribuir para o levantamento de informações relevantes da rede de parceiros sexuais, possibilitando uma melhor compreensão sobre a disseminação da infecção de HIV nessa população.

A utilização de uma combinação de aspectos de comportamentos de risco individuais e de parceiros sexuais para a construção de um índice pode contribuir para uma melhor avaliação do risco de contaminação por HIV. Na prática, esse índice pode ajudar em uma melhor avaliação do risco de se infectar pelo HIV na população de HSH e assim apoiar na elaboração de estratégias específicas de prevenção e tratamento para HIV/ Aids direcionadas a essa população. Além disso, a proposta de uso de um índice de risco para infecção por HIV também teve como foco a utilização prática na rotina de serviços de saúde, podendo ser empregado, por exemplo, para a triagem de indivíduos de maior risco.

Os resultados indicam que a proposta de desenvolvimento de um índice de risco para infecção por HIV pode ser relevante para identificar HSH com maiores probabilidades de contraí-lo. O grande diferencial da utilização do índice construído foi a incorporação de informações da rede de parceiros sexuais e das interações entre ego e alter, em detrimento do uso exclusivo de carac- 
terísticas individuais (pessoais) de risco, método normalmente utilizado em pesquisas comportamentais. Entretanto, por causa dos desafios e das limitações encontrados na condução do estudo, o que resultou em uma amostra pequena e diferenciada da população de $\mathrm{HSH}$, acredita-se que sejam necessários outras pesquisas para uma melhor validação do uso do escore. Assim seria possível complementar ou aperfeiçoar o método proposto neste estudo.

\section{Colaboradores}

RMC Torres participou da concepção, do desenho do estudo, da análise e interpretação dos dados e da redação final. Ils Bastos e MFC Gomes participaram da concepção e do desenho do estudo, da análise dos dados e da revisão final do manuscrito. RI Moreira participou do desenho do estudo, da coleta dos dados e da revisão final do artigo. ARS Périssé trabalhou na concepção, no desenho do estudo, na análise e interpretação dos dados e na revisão final. MM Cruz participou da concepção, da interpretação dos dados e da revisão final do manuscrito.

\section{Agradecimentos}

À Coordenação de Aperfeiçoamento de Pessoal de Nível Superior (CAPES). Aos grupos Arco-Íris e Pela VIDDA e à Estratégia de Saúde da Família Lapa pela disponibilidade e o apoio à realização da pesquisa. À Secretaria de Estado de Saúde do Rio de Janeiro pela disponibilização de insumos e apoio à realização da pesquisa. Ao Programa de Doutorado em Saúde Pública da Escola Nacional de Saúde Pública Sergio Arouca (ENSP/ Fiocruz). Ao Laboratório de Pesquisa Clínica em DST e AIDS do Instituto Nacional de Infectologia Evandro Chagas (INI/Fiocruz) pela parceria no desenvolvimento e implementação do estudo. 


\section{Financiamento}

O presente trabalho recebeu financiamento do Ministério da Saúde por meio do acordo de cooperação técnica entre o Departamento de Doenças Sexualmente Transmissíveis (DST), AIDS e Hepatites Virais da Secretaria de Vigilância em Saúde do Ministério da Saúde e o Escritório das Nações Unidas sobre Drogas e Crime (UNODC).

\section{Referências}

1. Baptista CJ, Dourado I, Andrade TM, Brignol S, Bertoni N, Bastos FI. HIV prevalence, knowledge, attitudes, and practices among polydrug users in Brazil: a biological survey using respondent driven sampling. AIDS Behav 2017; 22(7):2089-2103.

2. Grinberg G, Giron LB, Knoll RK, Galinskas J, Camargo M, Arif MS, Samer S, Janini LMR, Sucupira MCA, Diaz RS. High prevalence and incidence of HIV-1 in a counseling and testing center in the city of Itajaí, Brazil. Braz J Infect Dis 2015; 19(6):631-635.

3. Szwarcwald CL, Souza Júnior PRB, Pascom ARP, Ferreira Junior OC. Results from a method for estimating HIV incidence based on the first CD4 count among treatment-naïve cases: Brazil, 2004-2013. J AIDS Clin Res 2016; 7:11.

4. UNAIDS. How AIDS changed everything. MDG 6: 15 years, 15 lessons of hope from the AIDS Response. Joint United Nations Programme on HIV/AIDS. World Health Organization; 2015.

5. Cavalcanti AMS, Brito AM, Salustiano DM, Lima KO, Silva SP, Lacerda HR. Recent HIV infection rates among HIV positive patients seeking voluntary counseling and testing centers in the metropolitan region of Recife-PE, Brazil. Braz J Infect Dis 2012; 16(2):157163.

6. Castro CA, Grinsztejn B, Veloso VG, Bastos FI, Pilotto JH, Morgado MG. Prevalence, estimated HIV-1 incidence and viral diversity among people seeking voluntary counseling and testing services in Rio de Janeiro, Brazil. BMC Infect Dis 2010; 10(1):224.

7. Rocha GM, Kerr LRFS, Kendall C, Guimaraes MDC. Risk behavior score: a practical approach for assessing risk among MSM in Brazil. Braz J Infect Dis 2018; 22(2):113-122.

8. Szwarcwald CL, Ferreira Júnior O da C, Brito AM, Luhm KR, Ribeiro CEL, Silva AM, Cavalcanti MAS, Ito TS, Raboni SM, Souza Júnior PRB, Pereira GFM. Estimation of HIV incidence in two Brazilian municipalities, 2013. Rev Saude Publica 2016; 50:55.

9. Torres RMC, Cruz MM, Perissé ARS, Pires DRF. High HIV infection prevalence in a group of men who have sex with men. Braz J Infect Dis 2017; 21(6):596-605.

10. Cunha CB, De Boni RB, Guimarães MRC, Yanavich C, Veloso VG, Moreira RI, Hoagland BRS, Grinsztejn BGJ, Friedman RK. Unprotected sex among men who have sex with men living with HIV in Brazil: a crosssectional study in Rio de Janeiro. BMC Public Health 2014; 14

11. Silva AP, Greco M, Fausto MA, Greco DB, Carneiro M. Risk factors associated with HIV infection among male homosexuals and bisexuals followed in an open cohort study: Project Horizonte, Brazil (1994-2010). PLoS ONE 2014; 3;9(10):e109390.

12. Szwarcwald CL, Andrade CLT, Pascom ARP, Fazito E, Pereira GFM, Penha IT. HIV-related risky practices among Brazilian young men, 2007. Cad Saude Publica 2011; 27(Supl. 1):s19-s26.

13. Yi S, Tuot S, Chhoun P, Pal K, Tith K, Brody C. Factors associated with inconsistent condom use among men who have sex with men in Cambodia. Newman PA, editor. PLoS ONE 2015; 10(8):e0136114. 
14. Amirkhanian YA. Social networks, sexual networks and HIV risk in men who have sex with men. Curr HIV/AIDS Rep 2014; 11(1):81-92.

15. Brignol S, Dourado I, Amorim LD, Kerr LRFS. Vulnerability in the context of HIV and syphilis infection in a population of men who have sex with men (MSM) in Salvador, Bahia State, Brazil. Cad Saude Publica 2015; 31(5):1035-1048.

16. Janulis P, Phillips II G, Birkett M, Mustanski B. Sexual networks of racially diverse young msm differ in racial homophily but not concurrency. J Acquir Immune Defic Syndr 2017; 77(5):459-466.

17. Mustanski B, Birkett M, Kuhns LM, Latkin CA, Muth $\mathrm{SQ}$. The role of geographic and network factors in racial disparities in HIV among young men who have sex with men: an egocentric network study. AIDS Behav 2015; 19(6):1037-1047.

18. Périssé AR, Langenberg P, Hungerford L, Boulay M, Charurat M, Schechter M, Blattner W. Egocentric network data provide additional information for characterizing an individual's HIV risk profile. AIDS 2010; 24(2):291-298.

19. Rodriguez-Hart C, Liu H, Nowak RG, Orazulike I, Zorowitz S, Crowell TA, Baral SD, Blattner W, Charurat $\mathrm{M}$. Serosorting and sexual risk for HIV infection at the ego-alter dyadic level: an egocentric sexual network study among MSM in Nigeria. AIDS Behav 2016; 20(11):2762-2771.

20. Tieu H-V, Nandi V, Hoover DR, Lucy D, Stewart K, Frye V, Cerda M, Ompad D, Latkin C, Koblin BA. Do sexual networks of men who have sex with men in New York city differ by race/ethnicity? AIDS Patient Care STDs 2016; 30(1):39-47.

21. Doherty IA, Padian NS, Marlow C, Aral SO. Determinants and consequences of sexual networks as they affect the spread of sexually transmitted infections. $J$ Infect Dis 2005; 191(Suppl. 1):s42-s54.

22. Périssé ARS, Nery JAC. The relevance of social network analysis on the epidemiology and prevention of sexually transmitted diseases. Cad Saude Publica 2007; 23(Supl. 3):s361-s369.

23. Rothenberg RB, Potterat JJ, Woodhouse DE, Muth SQ, Darrow WW, Klovdahl AS. Social network dynamics and HIV transmission. AIDS 1998; 12(12):1529-1536.

24. Neaigus A, Friedman S, Goldstein M, Ildefonso G, Curtis R, Jose B. Using dyadic data for a network analysis of HIV infection and risk behaviors among injecting drug users. NIDA Res Monogr 1995; 151:2037.

25. Mattson CL, Campbell RT, Karabatsos G, Agot K, Ndinya-Achola JO, Moses S, Bailey RC. Scaling sexual behavior or "sexual risk propensity" among men at risk for HIV in Kisumu, Kenya. AIDS Behav 2010; 14(1):162-172.

26. Menza TW, Hughes JP, Celum CL, Golden MR. Prediction of HIV acquisition among men who have sex with men. Sex Transm Dis 2009; 36(9):547-555.

27. Trotter RT. Friends, relatives, and relevant others: conducting ethnographic network studies. In: Schensul JJ, LeCompte MD, Trotter RT, Cromley EK, Singer M. Mapping Social Networks, Spatial Data, \& Hidden Populations. California: AltaMira Press; 1999. p. 1-50.
28. Miller WM, Buckingham L, Sanchez-Dominguez MS Morales-Miranda S, Paz-Bailey G. Systematic review of HIV prevalence studies among key populations in Latin America and the Caribbean. Salud Publica Mex. 2013; 55(Suppl. 1):S65-78.

29. Campbell KE, Lee BA. Name generators in surveys of personal networks. Soc Netw 1991; 13(3):203-221.

30. Perisse ARS, Langenberg P, Hungerford L, Boulay M, Charurat M, Schechter M, Blattner W. The use of supplementary techniques to increase recall of sex partners in a network-based research study in Rio de Janeiro, Brazil. Sex Transm Dis 2008; 35(7):674-678.

31. Chen Y-H, McFarland W, Raymond HF, Scott HM, Vittinghoff E, Porco TC. Distribution of behavioral patterns before infection among San Francisco men who have sex with men newly infected with HIV in 2014. J Acquir Immune Defic Syndr 2017; 75(5):528534.

32. Goodreau SM, Carnegie NB, Vittinghoff E, Lama JR, Sanchez J, Grinsztejn B, Koblin BA, Mayer KH, Buchbinder SP. What drives the US and Peruvian HIV epidemics in men who have sex with men (MSM)? PloS One 2012; 7(11):e50522.

33. Hakim A, Patnaik P, Telly N, Ballo T, Traore B, Doumbia S, Lahuerta M. High prevalence of concurrent male-male partnerships in the context of low human immunodeficiency virus testing among men who have sex with men in Bamako, Mali. Sex Transm Dis 2017; 44(9):565-570.

34. Hamilton DT, Morris M. The racial disparities in STI in the U.S.: concurrency, STI prevalence, and heterogeneity in partner selection. Epidemics 2015; 11:56-61

35. Li R, Wang H, Pan X, Ma Q, Chen L, Zhou X, Jiang T, He L, Chen J, Zhang X, Luo Y, Xi S, Lv X, Xia S. Prevalence of condomless anal intercourse and recent HIV testing and their associated factors among men who have sex with men in Hangzhou, China: a respondent-driven sampling survey. PloS One 2017; 12(3): $\mathrm{e} 0167730$

36. Hosmer DW, Lemeshow S, Sturdivant RX. Applied logistic regression. New Jersey: Wiley; 2013.

37. Brignol SMS, Dourado I, Amorim LD, Miranda JGV, Kerr LRFS. Social networks of men who have sex with men: a study of recruitment chains using Respondent Driven Sampling in Salvador, Bahia State, Brazil. Cad Saude Publica 2015; 31(Supl. 1):170-181.

38. Scott J. Social network analysis: a handbook. London: Sage Publications; 2000.

39. Hickson DA, Mena LA, Wilton L, Tieu H-V, Koblin BA, Cummings V, Latkin C, Mayer KH. Sexual networks, dyadic characteristics, and HIV acquisition and transmission behaviors among black men who have sex with men in 6 US cities. Am J Epidemiol 2017; 185(9):786-800.

40. Hedberg EC. Dyad vs. network effects: modeling relationships in personal networks using contextual effects. Soc Sci Res 2017; 63:339-355.

41. Rinehart DJ, Al-Tayyib AA, Sionean C, Whitesell NR, Dreisbach S, Bull S. Assessing the theory of gender and power: HIV risk among heterosexual minority dyads. AIDS Behav 2018; 22(6):1944-1954. 
42. Shrier LA, Schillinger JA, Aneja P, Rice PA, Batteiger BE, Braslins PG, Orr DP, Fortenberry D. Depressive symptoms and sexual risk behavior in young, chlamydia-infected, heterosexual dyads. J Adolesc Health 2009; 45(1):63-69.

43. MacCarthy S, Reisner S, Hoffmann M, Perez-Brumer A, Silva-Santisteban A, Nunn A, Bastos L, Vasconcellos MTL, Kerr L, Bastos FI, Dourado I. Mind the gap: implementation challenges break the link between HIV/ AIDS research and practice. Cad Saude Publica 2016; 32(10):e00047715.

44. Magnani R, Sabin K, Saidel T, Heckathorn D. Review of sampling hard-to-reach and hidden populations for HIV surveillance. AIDS 2005; 19(Suppl. 2):s67-s72.

Artigo apresentado em 14/02/2019

Aprovado em 05/01/2020

Versão final apresentada em 07/01/2020

Editores-chefes: Romeu Gomes, Antônio Augusto Moura da Silva 